\title{
Hand rejuvenation with CaHA guided by ultrasound
}

\begin{abstract}
Volumizing the dorsal hand is one of the anatomical regions most frequently requested during the last decade. Calcium hydroxyapatite is the material used for this procedure since its beginning, due to its several benefits. However, despite the consensus among doctors of the dermal filler to be applied, the same does not happen to the chosen technique. This variation in methods is due to the divergence between anatomical studies in this region. Through this, we review the literature in order to establish the best method and confirming the correct plan through ultrasound to inject this material.
\end{abstract}

Volume 4 Issue 2 - 2020

\author{
Barbara Poggi \\ Department of dermatology, Hospital Naval Marcílio Dias, Rio \\ de Janeiro, Brazil
}

Correspondence: Barbara Poggi, Department of dermatology, Hospital Naval Marcílio Dias, Rio de Janeiro, Brazil,

Email baby_poggi@hotmail.com

Received: April 22, 2020 | Published: April 30, 2020

\section{Background}

The effectiveness of the use of calcium hydroxyapatite for hand rejuvenation is well documented in the literature, but studies on anatomy are conflicting. The aim of the present study is to show the application of calcium hidroxiapatite in dorsal hand by distal proximal technique and concomitant visualization by USG $15 \mathrm{Mhz}$, combined to literature to define, ensuring the injection safety in the correct plan.

\section{Introduction}

The dorsal hand's filler application has been one of the anatomical regions that most increased the last decade. ${ }^{1,2}$ Many material of dermal fillers can be injected in this region as autologous fat, polyL-latic, carboxy methyl cellulose, hyaluronic acid (HA) and calcium hydroxylapatite (CaHA). ${ }^{1,3}$ Currently, the lastter is the only dermal filler approved by the FDA for use in hands ${ }^{4}$ and has particullary increased attention due to its several benefits like: achievieving the desired effect ate the first injection ${ }^{4,5}$ and which noteworthy remained through 12 months postenrol ment after it. ${ }^{4,2}$ Another advantage inherent of this two part gel is its own capacity of concealing the bluish tint associated with visible veins and tendons in the hands owing to its opacity. ${ }^{4,5}$ The CaHA can be injected in dorsal hand by needle or cannula and by four different techniques: bolus, tenting, proximal-todistal fanning and distal-to-proximal single-line technique ${ }^{1}$ or even some of them combinated. ${ }^{6}$ Thus, we proposed the use of ultrasound to study thoroughly the dorsal hand anatomy, because the histology lacks of contiguous tissue planes's elucidation and the previously anatomic dissection studies didn't perfect represented the changes in tissue volume of the dorsal hand because of unpredictable postmor. ${ }^{6,7}$ Ultrasound has been used in dermatology since 1970 but it has been popularized nowadays with the hight frequency machines. The ones with frequency bigger than $15 \mathrm{MHz}^{7}$ have low penetration, ${ }^{7}$ and therefore, can show the thickness of the layers and their interconnections. ${ }^{2}$ Because of it, we believe that it is the most reliable exam to recognize structures of the dorsum at the same moment of the injection. Dermatology adjuvant ultrasound imaging has increased progressively and newest studies suggest that its anatomical results significantly match to the previous literature data. ${ }^{8}$ Another advantage of this imaging method is the analysis during the injection which allows to prove reaching of the whished plan.

\section{Methods}

Two women aged 46 and 48 had their dorsal hands treated for cosmetic hand volumizing procedures using a calcium hydroxylapatite product (Radiesse; Merz North America, Raleigh, N.C.) in a single center (Clínica Bravo, Rio de Janeiro, Brazil). All of them presented clinical signs of moderate intense loss of soft tissue on the dorsum of the hands, interosseous muscle atrophy, with wrinkles, tendons and veins being visible. First of all, we diluted $1,5 \mathrm{~mL}$ of $\mathrm{CaHa}$ at $6 \mathrm{~mL}$ of $2 \%$ lidocaine, with in addition to reduces the pain, decreases its viscosity, requiring a smaller extrusion force and making it more spreadable and less palpable. ${ }^{5,3,9}$ The individuals underwent two photographs before and after the procedure and the ultrasound imaging, which was performed by a butterfly ultrasound in B-mode was became during CaHA application. The same dermatologist applied CaHA into superficial dorsal lamina in retrograde movement through proximal-to-distal fanning technique in only one proximal incision in each dorsum with $22 \mathrm{G}$ cannula at a single session. The cannula was inserted right below the skin after a hope was opened by a $21 \mathrm{G}$ needle and advanced within the subdermal plane until it arrives the metacarpophalangeal joints. During the same time, the skin lackers were visualized by ultrasound.

\section{Results}

Our study showed that the visualization of the anatomy of the dorsum of the hands by ultrasound is a great interest to Cosmetic Dermatology, since the structures can be clearly analyzed by the examining physician, giving greater security in the completion of hand filling with CaHa.

During the procedure, the filler injection was observed as a hyperechogenic with shadow image at superficial dorsal lamina through proximal-to-distal fanning technique and the layers was correctly visualized, ensuring a correct plane application.

As we can see in the photo bellow, we observed the layered arrangement of the dorsum of the hand, the skin surface, dorsal superficial lamina, dorsal superficial fascia, dorsal intermediate lamina (DIL), dorsal intermediate fascia, the dorsal deep fascia which is strongly connected to the DIL, forming a compartment that is a housing to the extensor tendon of the wirst. 
All fascias are shown in white colors, cause they are hipereconegic The laminas are colored in black, wich is hypoecogenic. With the Doppler technique we can also visualize the dorsal superficial venous, wich is colored in blue and the artery, colored in red. Like most cases described, there were no changes in hand function or other significant side effects in our patients. ${ }^{4}$

\section{Discussion}

Old studies analyzed suggest skin tenting to separate it from underlying vasculature and tendons and then the needle was advanced between the subcutaneous layer and the superficial fascia. ${ }^{4,10}$ However, the newest ones, alert about elevated risk taken on in this technique. Cotofana and col suggest that this skin lifting, due to strong fibrous underlying connections, elevates additionally below fascial and fatty layers, including the ones which contain the veins and sensory nerves. ${ }^{2}$ In their study, they proceeded anatomical dissections and imaging exams of hands dorsal side and concluded that it contains three different laminae: superficial, intermediate, and deep, propped by its respective fascia. Because of the absence of major veins and sensory nerves in the first one, they suggest that CaHA injections should be applied in that plan, which more specifically distances less than $1 \mathrm{~mm}$ from the skin surface (when measured by ultrasound). Moreover, this layer provides a free gliding space for the proximalto-distal fanning technique using a cannula, differing of its underlying fascia, the dorsal superficial fascia, which distances from the skin surface of $0.98 \pm 0.16 \mathrm{~mm}$ (when measured by ultrasound) and delimits the beginning of the dorsal veins and the dorsal sensory branches of the radial and ulnar nerves. They stand up for the injection by proximal-todistal fanning technique because of cannula's movement suggesting that CaHA should go along it first retrograde and after antegrade paths and also by variation of anatomy in distal part adducing that in dorsal distal third the layered arrangement isn't clearly as it is in the proximal two-thirds. ${ }^{1}$ They add that dorsal intermediate fascia is closely linked to dorsal deep lamina constituting fibrous canals for the extensor tendons, a theory that resembles some articles presents at literature. Another study based on fresh cadaveric dissections and imaging exams describes it as "a lot of tiny multidirectional brous septa giving a 3- dimensional (3D) framework similar to the "walls" of a sponge around its tunnels". ${ }^{2}$ Agreeing to them, Lefebvre-Vilardebo and col defend that the CaHA injecton should take place in fascial layer but the add that due to it extremely thickness at the time of a rejuvenation (no more than $1 \mathrm{~mm}$ ) performing this prodedure in a blind manner has elevated risc of injecting the product in a underpredictable place, what they show in the ultrasound images. ${ }^{2}$

\section{Conclusion}

Despite literature is contradictory, our ultrasound analysis matched to several studies, implying a layered arrangement in dorsum of the hand, between the epidermis and the deep fascia and the best method to rejuvenation this anatomical segment being the injection of CaHA subdermal, using a cannula, from proximal to distal. This definition is important because this body's part has the biggest thinness at the time of rejuvenation which, in combination of the its extreme mobility, requires a special study. ${ }^{2}$ Lately, the use of High-frequency ultrasound $(20-100 \mathrm{MHz})^{7,11}$ is an adequate tool for objective quantification of the best method and the results of CaHA injection.

\section{Conflits of interest}

The author declares that there is no conflicts of interests.

\section{Acknowledgments}

None.

\section{Funding}

None.

\section{References}

1. Frank K, Koban K, Targosinski S, et al. The Anatomy behind Adverse Events in Hand Volumizing Procedures: Retrospective Evaluations of 11 Years of Experience. Plast Reconstr Surg. 2018;141(5):650e-662e.

2. Lefebvre-Vilardebo M, Trevidic P, Moradi A, et al. Bucay VW. Hand: Clinical anatomy and regional approaches with injectable fillers. Plast Reconstr Surg. 2015;136(5):258S-275S.

3. Medical B, Mateo S. Comparison of the Rheological Properties of Viscosity and Hydroxylapatite and Hyaluronic Acid. Dermatol Surg. 2010;1859-1865.

4. Goldman MP, Moradi A, Gold MH, et al. Calcium Hydroxylapatite Dermal Filler for Treatment of Dorsal Hand Volume Loss: Results From a 12-Month, Multicenter, Randomized, Blinded Trial. Dermatol Surg. 2018;44(1):75-83.

5. Edelson KL. Hand recontouring with calcium hydroxylapatite (Radiesse)®. J Cosmet Dermatol. 2009;8(1):44-51.

6. Zhou J, Xie Y, Wang WJ, et al. Hand rejuvenation by targeted volume restoration of the dorsal fat compartments. Aesthetic Surg J. 2018;38(1):92-100.

7. Barcaui EDO, Carlos A, Carvalho P, et al. Study of the skin anatomy with high-frequency (22 MHz)ultrasonography and histological correlation. Radiologia Brasileira. 2015;48(5):324-329.

8. Bidic SM, Hatef DA, Rohrich RJ. Dorsal hand anatomy relevant to volumetric rejuvenation. Plast Reconstr Surg. 2010;126(1):163-168.

9. Busso M, Applebaum D. Hand augmentation with Radiesse ${ }^{\circledR}$ (Calcium hydroxylapatite). Dermatol Ther. 2007;20(6):385-387.

10. Bertucci V, Solish N, Wong M, et al. Evaluation of the Merz Hand Grading Scale After Calcium Hydroxylapatite Hand Treatment. Dermatol Surg. 2015;41:S389-396.

11. Tedeschi A, Lacarrubba F, Micali G. Mesotherapy with an Intradermal Hyaluronic Acid Formulation for Skin Rejuvenation: An Intrapatient, Placebo-Controlled, Long-Term Trial Using High-Frequency Ultrasound. Aesthetic Plast Surg. 2014;39(1):129-133. 